SULL' LSO DELL' ELETTROME'RRO NELLO STUDIO COMPIUTO DELLE COPPIE VOL'TAICHE A ClRCUITO CHIUSO; NOTA DEI DOTt. GIOVANNI GUGLIELMO.

Fra le numerose applicazioni dell'elettrometro a quadranti, won è delle meno importanti quella fattane alla misura delle, forze elettromotrici delle coppie a circuito aperto. che così riducesi alla massima semplicità eliminando inoltre parecchie cause d'errore.

$\grave{\mathrm{E}}$ oggetto del presente laroro il mostrare como coll' uso dell' elettrometro venga semplificato anche 10 studio delle coppie a circuito chiuso, giacchè senza alcun altro strumento di misura, ed assai rapidamente, si ottiene la differenza di potenziale di due punti del circuito, e la resistenza interna della coppia, con che, se è nota la resistenza esterna, è determinata anche la forza elettromotrice.

La determinazione della resistenza interna si fa col metodo del Mance; se nella chiusura del reoforo AC ( Tav. IV, fig. 1) non si produce alcuna variazione nell' intensita della corrente che percorre il reoforo $\mathrm{BD}$ (ossia nel nostro caso non varia la differenza di potenziale dei punti $B, D$ che comunicano coi due quadranti dell' elettrometro) sarà come è noto $\frac{\mathrm{R}_{1}}{\mathrm{R}}=\frac{r_{1}}{r}, \theta$, se $\mathrm{R}_{4}=\mathrm{R}$, sarà $r=r_{1}$ resistenza interna della coppia. Inoltre sara la forza elettromotrice

$$
\mathrm{E}=\mathrm{D} \frac{2 \mathrm{R}+2 r}{\mathrm{R}+r}=2 \mathrm{D}
$$

se $D$ è la differenza di potenziale di detti punti $B, D$.

$\dot{\mathrm{E}}$ facile giungere a questa relazione, ed ottenere inoltre le condizioni di sensibilità.

Lal differenza di potenziale dei punti $B, D$ nor Ar snorta i.

$$
\mathrm{D}(\mathrm{BD})=\mathrm{E} \frac{\mathrm{R}+r}{\mathrm{R}+\mathrm{R}_{1}+r+r_{1}} .
$$


Se si chiude AC, che supporremo abbia una resistenza $p$, sarà

$$
\mathrm{D}_{1}(\mathrm{BD})=\mathrm{F} \frac{r+\frac{\mathrm{R}}{\mathrm{R}+\mathrm{R}_{1}} x}{r+r_{1}+x},
$$

dore $x$ è la resistenza totale dei due tratti

quindi

$$
A B C, A C,=\frac{\left(R+R_{1}\right) p}{R+R_{1}+p}
$$

$$
D_{1}(\mathrm{BD})=\mathrm{E} \frac{\left(\mathrm{R}+\mathrm{R}_{4}+p\right) r+\mathrm{R}_{p}}{\left(\mathrm{R}+\mathrm{R}_{1}+\rho\right)\left(r+r_{1}\right)+\left(\mathrm{R}+\mathrm{R}_{1}\right) p}
$$

e la variazione per la chiusura di AC sarà :

(a) $\left\{\begin{array}{c}\mathrm{D}_{1}(\mathrm{BD})-\mathrm{D}(\mathrm{BD}) \\ =\mathrm{E} \frac{\left(\mathrm{R}+\mathrm{R}_{1}+\rho\right) r+r \rho}{\left(\mathrm{R}+\mathrm{R}_{1}+\rho\right)\left(r+r_{1}\right)+\left(\mathrm{R}+\mathrm{R}_{1}\right) \rho}-\mathrm{E} \frac{\mathrm{R}+r}{\mathrm{R}+\mathrm{R}_{1}+r+r_{1}} \\ =\frac{\left(\mathrm{R}+\mathrm{R}_{1}\right)\left(\mathrm{R}_{1} r-\mathrm{R} r_{1}\right)}{\left[\left(\mathrm{R}+\mathrm{R}_{1}\right)\left(r+r_{1}\right)+\rho\left(\mathrm{R}+\mathrm{R}_{1}+r+r_{1}\right)\right]\left(\mathrm{R}+\mathrm{R}_{1}+r+r_{1}\right)} \mathrm{E}\end{array}\right.$ che sarà nulla per

ossia se

$$
R_{1} r-R r_{1}=0
$$

$$
\frac{\mathrm{R}}{\mathrm{R}_{\mathrm{i}}}=\frac{r}{r_{\mathrm{i}}} .
$$

Riguardo alle condizioni di sensibilità, vedesi anzitıtto che $D_{1}-D$ è proporzionale ad $E, e$ cresce col decrescere di p.

$$
\text { Per } \rho=0, \frac{D_{1}-D}{\mathrm{E}}=\frac{\mathrm{R}_{1} r-\mathrm{R} r_{1}}{\left(\mathrm{R}+\mathrm{R}_{1}+r+r_{1}\right)\left(r+r_{1}\right)},
$$

e dividendo per $R_{1}$, se $\frac{R}{R_{4}} m$, sarà :

$$
\frac{\mathrm{D}_{1}-\mathrm{D}}{\mathrm{E}}=\frac{r-m r_{1}}{\left(1+n+\frac{r+r_{1}}{\mathrm{R}_{1}}\right)\left(r+r_{1}\right)} \text {, }
$$


968

dove vedesi che $D_{1}-1$ cresce col decrescere di $\frac{r+r_{1}}{R}$ lentamente prima e poi rapidamente quando $R$, sia molto minore di $r+r_{1}$. Un risultato simile si ayrebbe per $R$, o s? si fosse operato sull' espressione generale $(a)$.

Finalmente per vedere l'influenza del rapporto di $R$ ad $R_{1}$, sia $R+R_{1}=K$ costante, $\frac{R}{R_{1}}=m$ ossia

$$
\mathrm{R}=\frac{\mathrm{Km}}{1+n}, \mathrm{R}_{1}=\frac{\mathrm{K}}{1+m} \text {, }
$$

e $r+r_{1}=k$ costante $\theta \frac{r}{r_{1}^{\prime}}=m, r_{1}=r_{1}^{\prime}$ valore giusto $\pm \mathrm{ul}$ errore relativo costante $\varepsilon r_{\mathfrak{1}}^{\prime}$ ossia :

$$
r=\frac{k m}{1+m \pm \varepsilon}, \quad r_{1}=\frac{k(1 \pm \varepsilon)}{1+m \pm \varepsilon} .
$$

sostituendo, nella (a) non resta di variabile che il fattore

$$
\begin{aligned}
& \mathrm{R}_{1} r-\mathrm{R} r_{1}=\mathrm{R}_{1} r_{1}\left(\frac{m}{1 \pm \varepsilon}-m\right)= \pm \varepsilon m \mathrm{R}_{1} r_{1}= \pm \varepsilon \mathrm{R} r \\
& =\mp \varepsilon \frac{\mathrm{K} m}{1+m} \cdot \frac{k(1 \pm \varepsilon)}{1+m \pm \varepsilon}=\text { Grossimadingte } \mathrm{a} \mp \varepsilon \mathrm{K} k \frac{m}{(1+m)^{2}} .
\end{aligned}
$$

Differenziando ed uguagliando a zero, o con semplice artificio algebrico, trovasi che si ha il massimo per $m=1$.

In questo caso e per $p=0$ è :

$$
\frac{\mathrm{D}^{\prime}-\mathrm{D}}{\mathrm{E}}=\frac{\mathrm{R}\left(r-r_{1}\right)}{\left(2 \mathrm{R}+r+r_{1}\right)\left(r+r_{1}\right)} \text {. }
$$

Se p. es. nel caso della coppia Poggendorff i: $R=100$, $r=0.20, r_{1} \rightarrow 0.21$ sarà :

$$
\frac{\mathrm{D}_{1}-\mathrm{D}}{\mathrm{H}}=\frac{100 \cdot 0,41}{200,41 \cdot 0,41}=\frac{1}{82} \text {. }
$$

ossia lo spostamento dell' ago sarà "/8z della deviazione prodotta dalla coppia, e se questo in un caso punto favorevole 
è di 100 divisioni, lo spostamento sarà di ${ }^{100}{ }_{89}$ di divisiono c permetterà di apprezzare il millesimo di unità Siemens, sensibilità più che sufficiente per una quantità non troppo costante. Se fosse $R=1$ sarebbe $\frac{D^{\prime}-D}{E}=\frac{1}{100}$, ma se $r=4,2$, $r_{1}=4$ sarebbe $\frac{\mathrm{D}^{\prime}-\mathrm{D}}{\mathrm{E}}=\frac{1}{418}$.

S' è visto come nel nostro caso assai pianamente si giunga alla espressione generale di $D^{\prime}-D$ e. se ne faccia la discussione, che p. es. nella memoria del Lodge non è scevra di qualche complicazione ed artificio. Graficamente si ottiene anche maggior semplicità.

Rappresenti DD (fig. 2) la resistenza del circuito totale e sia $\mathrm{DA}=r$ ec. Supponiamo che la forza elettromotrice si trovi alla estremità della resistenza $r$, della coppia, e si tenga $D$ in comunicazione col suolo, cosicchè rappresenti DdD l'andamento dei potenziali nel circuito quando il reoforo $\mathrm{AC}$ è aperto. Si chiuda questo reoforo, la linea dei potenziali prender⿳亠 in $r$ ed $r_{1}$ un altro pendio e sarà rappresentata da $\mathrm{D} a^{\prime}$ e $\mathrm{D} c^{\prime}$, e l' andamento in $\mathrm{AC}$ potrà rappresentarsi con $a^{\prime} c^{\prime}$ se si tien conto che le resistenze rappresentate in quel tratto vanno moltiplicate pel fattore $\frac{A_{1} C}{A C}$. Vedesi che il potenziale è rimasto insariato pel solo punto $\mathrm{B}$ che divido $\mathrm{AC}$ nel rapporto di $r$ ad $r_{1}$, giacchè : $\frac{\mathrm{AB}}{\overline{\mathrm{BC}}}=\frac{a^{\prime} b}{b c^{\prime}}=\frac{a^{\prime} \mathrm{D}}{c^{\prime} \mathrm{D}}=\frac{\mathrm{DA}}{\overline{\mathrm{CD}}}$. Si vede inoltre che per una posizione attigua $B^{\prime}$ il potenziale avrà subito una variazione $\beta \beta^{\prime}$ che cresce col decrescere di $\rho$ che produce la diminuzione del pendio di $a^{\prime} c^{\prime}$. Decrescendo $\mathrm{R}+\mathrm{R}_{\mathrm{a}}$ p. es. nel caso di $p=0$ ossia $a^{\prime} c^{\prime}$ parallela a DD, la linea dei potenziali se il reoforo $\mathrm{AC}$ è chiuso, rimane la stessa. ma se AC è aperto (poichè il pendio in $r$ ed $r$, crescerà), potrà esser rappresentato da $\mathrm{D} a^{\prime \prime} c^{\prime \prime} d$ colla solita convenzione di moltiplicare le resistenze rappresentate nel tratto $\mathrm{AC}$ per un certo fattore. Ora vedesi clue l'angolo $a^{\prime} b a$ è divenuto $a^{\prime} b a$, e $\beta \beta^{\prime}$ è diminuito.

Finalmente, per scorgere l'infineuza del rapporto di R 
ad $R_{1}$ essendo $R+R_{1}$ ed $r+r_{1}$ costanti, osserviamo che il variare di $\frac{\mathrm{h}}{\mathrm{R}_{\mathrm{q}}}$ non fa che spostare parallelamente il tratto compreso fra le ordinate $\mathrm{A} a, \mathrm{C} c$, e spostare $\mathrm{B}$ rispetto ad $A$ e $C$, e $\beta \beta_{1}$ rimane costante se tale è $B B_{1}$ : ora prendendo $B_{1}$ invece di $\mathbf{B}$ commettiamo un errore relativo che dipende ugualmente dall'errore relativo di $R_{1}+\frac{\mathrm{BB}_{4}}{\overline{A B}_{1}}$ e da quello di $R_{1}$ che $\grave{e}-\frac{B B_{i}}{B_{1} C}$, ora il primo cresce rapidamente specialmente per $A B$ molto piccolo, montre il secondo decresce lentamente, quindi la stessa variazione del potenziale si avrà per errori tanto maggiori quanto più $A B \geq B C_{\text {, }}$ e quindi la sensibilità sarà massima per $\mathrm{AB}=\mathrm{BC}$.

Analogamente si potrebbe ragionare nella disposizione del ponte di Wheatstone.

$\mathrm{Nel} \mathrm{nostro} \mathrm{caso} \mathrm{è} \mathrm{inutile} \mathrm{o} \mathrm{almeno} \mathrm{non} \mathrm{necessaria} \mathrm{la} \mathrm{mo-}$ dificazione introdotta dal Lodye di interporre un condensatore nel reoforo $\mathrm{BD}$, giacchè essendo (entro certi limiti) la deviazione dell' ago proporzionale alla differenza di potenziale la sensibilità è la stessa qualunque sia la posizione dell'ago, nè lo strumento soffre per essere sottoposto ad una differenza di potenziale che non esce dai limiti di quelle ordinariamente osservate. Invece è indispensabile l'altra modificazione di interrompere il reoforo BD subito dopo la chiusura di $\mathrm{AC}$ per impedire l'influenza della variazione prodotta nella forza elettromotrice per la diminuita resistenza, influenza tanto più nociva nel nostro caso, in quanto il movimento dell'ago per effetto delle palettine immerse nell' acido solforico ò assai lento (specialmente per piccole rariazioni). Naturalmente nell'elettrometro di Branly tale influenza sarebbe meno nociva, ma sempre più che nel galvanometro.

Si eseguirono varie esperienze sulle coppie mono costanti piuttosto come prova del metodo, che con la pretesa di aggiungere o correggere i risultati gia ottenuti, tanto piu che per ristrettezza di tempo e poca quiete del locale nell' epocia 
delle esperienze, queste non sono state fatte nelle migliori condizioni.

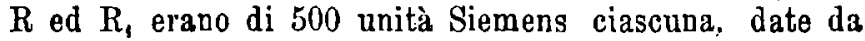
due reostati di Siemens e Halske; in $r$ era un reocordo ed in $r_{1}$ la coppia da studiare. I due punti $\mathrm{B}$ e $\mathrm{D}$ erano in comunicazione coi due quadranti di un elettrometro Mascart. costruito da Carpentier, il cui ago comunicava con un polo d'una pila Zamboni di 300 elementi, che aveva l'altro polo comunicante col piede dell' apparecchio e col suolo. La deviazione prodotta da una Daniell campione era di circa 54 divisioni quando la sospensione bifilare aveva il massimo allargamento e quindi era al minimo di sensibilità; nelle condizioni attuali era di circa 78. Evidentemente si potrebbe aumentare la sensibilità con una pila Zamboni più potente e con diminuire l'angolo della sospensione bifilare.

L'ago in ogni esperienza veniva disposto simmetricamente rispetto ai quadranti, e quindi quando questi comunicavano col suolo conservava assai prossimamente l'identica posizione, sia che l'ago comunicasse col suolo o col polo della pila Zamboni; la posizione dello zero era prossimamente la stessa in principio ed in fine della esperienza, come pure era la stessa la deviazione prodotta dalla Daniell canpione.

M' ero assicurato sperimentalmente (col noto mezzo di misurare la differenza di potenziale agli estremi d'un reostato in cui si fa variare la resistenza, mentre per mezzo di un altro roostato si mantiene costante la resistenza totale del circuito) della proporzionalità fra le differenze di potenziale e le deviazioni corrispondenti, entro i limiti delle esperienze.

La lettura delle deviazioni era fatta dapprima osservando su una scala divisa lo spostamento dell' immagine dell' orlo fisso d'una fessura luminosa; ma essendo troppo faticoso e poco preciso osservare in tal modo piccole variazioni, si fece la lettura coll'altro mezzo di osservare con un cannocchiale a reticolo gli spostamenti dell imagine della scala.

Per produrre la chiusura del reoforo $\mathrm{AC}$ e subito dopo la separazione dell' elettrometro dai punti $B, D$ questi comunicavano mediante l'asse di rotazione coi due bracci isolati 
272

$b, l$ (fig. 3) d'un commutatore a bilanciere simile a quello usato da kxuer nelle sto esperienze sulla polarizzazione, mentre i quadranti comunicavano colle colonnine pure isolate $b$, $d_{1}$. il punto A coll'astina isolata $a$ ed il punto $c$ con una astina metallica a manico isolante, colla quale toccando $a$ veniva chiuso il reoforo $\mathrm{AC}$ e sollevando il bilanciere si separavano $b$ e $d$ da $b_{1}$ e $d_{1}$ per cui rimaneva isolato l' elettrometro.

Potrebbe servire allo stesso scopo il tasto usato dal Lodge o tal quale, ma coll' arvertenza di isolarne bene le varie parti. oppure colla disposizione rappresentata dalla figura 4 .

F. da notare che nel chiudere AC l'elettromotro prende una carica corrispondente al nuovo circuito, e la conserva dopo la separazione da $\mathrm{BD}$, quindi colla nuova deviazione $\theta$ colla primitiva (quando il disperdimento fosse affatto trascurabile, come pare avrenga nell'elettrometro di Thomson e come certo non arveniva in questo di Mascart) si potrebbe calcolare senz' altro la resistenza della coppia e quindi la forza elettromotrice. Tuttaria è sempre preferibile produrre col variare di $r$ una variazione della deviazione in senso opposto e quindi ottenere per proporzione il valore assai più preciso della resistenza cercata.

Un inconveniente talora abbastanza nocivo, in questa come in ogni altra esperienza in cui si usi un commutatore, i lo sviluppo di elettricita per attrito o per altre cause accidentali (p. es. effetti d'induzione sui fili che conducono ai quadranti) nel moto del bilanciere e specialmente nello staccare $b d$ da $b_{1} d_{1} ;$ ma a ciò si rimedia facilmente aumentando la capacità elettrica dei due quadranti col porli in comunicazione colle armature d' un condensatore, sulle quali ripartendosi le piccole quantità di elettricità riescono insensibili (').

(1) Cin altro vantaggio dell'uso deJ condensatore è che pormette, con un solo contatto istantaneo, di ottenere prossimamente la deviazione corcata (con l'errore del disperdimento che in buone condizioni non è mai molto notevole), montro senza romtensatore, sia per essere la capacità dei quadranti variabile colia posizione dell'ago, o piuttosto per l'elettricitiz che va impiegata nol dare una certa carica ai sosterni occorrono parecchi contatti istantanei, o un contatto permanento per un jo' di tempo; ciò che non ha piccoli inconvenienti so Ja differonza di potenziale $\mathrm{i}$ variabile, cone in parecchi casi di polalizzazione $e$ forze elettronotrici. 
Un' altra causa d'errore $\dot{\theta}$ il disperdimento che tende a diminuire gli aumenti di deviazione e ad aumentare e talora prolungare indefinitamente le diminuzioni; pero questa non è molto sensibile quando si esperimenti in buone condizioni cioè l' acido solforico sia posto da un po' di tempo ('), l'ambiente non sia troppo umido ec. e ciò anche per l'elettrometro Mascart che non pare sia a questo proposito nelle migliori condizioni.

Del resto, è facile eliminare completamente questo inconveniente usando una disposizione analoga a quella del Lodge, cioè interponendo fra i quadranti e le armature del condensatore in ciascun filo deil'elettrometro un altro condensatore e riduceudo prima di chiudere $A C$ i due quadranti allo zero. Ma non essendo comodo l'usare tre condensatori, è sufficiente uno solo, quando uno dei quadranti si ponga in comunicazione col suolo, e si interponga il condensatore nel tilo che conduce all' altro quadrante. Lo stesso effetto s'arrebbe anche ponendo $\mathrm{A}$ in comunicazione coll' ago, $\mathrm{C}$ col suolo, ed i due quadranti coi due poli d'una piccola pila a gran tensione il cui mezzo comunica col suolo. Evidentemente la Zamboui non si presta a tale uso perchè non è facile porne il punto medio in comunicazione col suolo.

(1) Se peri, 1' acido sulforico fosse stato posto da troppo tempo, prubalisilmente prr cansa di pulviscolo, perde in fluidita, ed ogui piì pircola trepidazione del s;t trno viene traşmessa al]' ago, the cosi non rimane mai fermo. 
274

Ecco ora i risultati di varie esperienze:

Coppia Bunsen ad acido cromico.

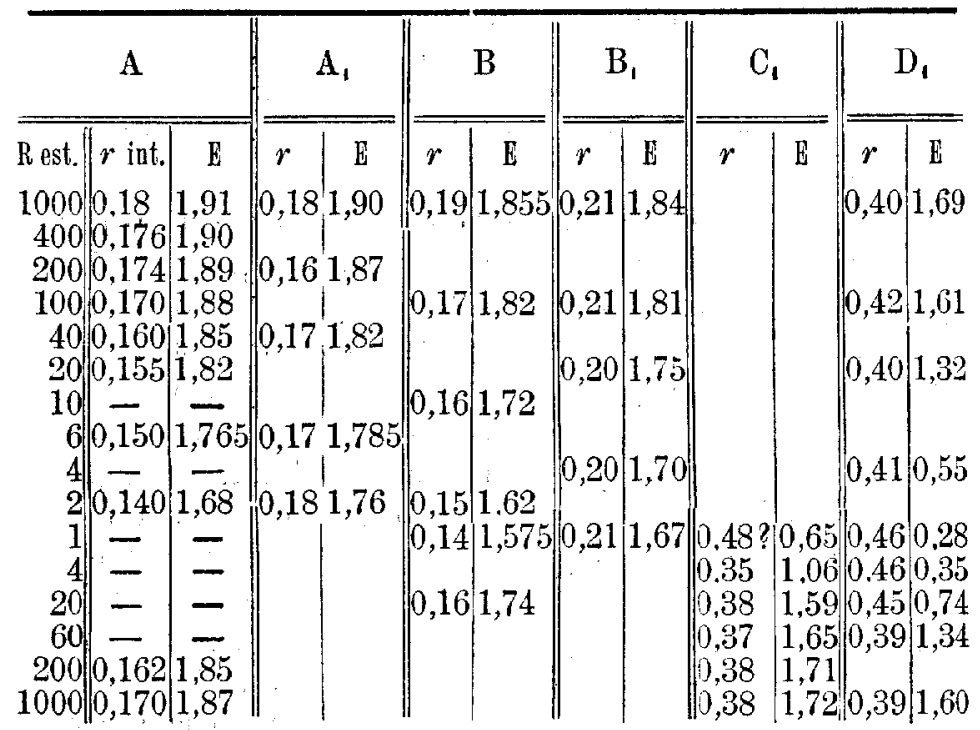

Le resistenze sono espresse in unità Siemens; le forze elettromotrici $\mathrm{E}$ prendendo per unità quella della Daniell. In generale dopo determinata la resistenza della coppia, si lasciavano scorrere 5 minuti o più prima di leggere la deviazione.

Le serie A, A, furono fatte con liquidi nuovi.

La serie B 16 ore dopo la A; essendo stato in questo intervallo il circuito chiuso con una resistenza $=1000 \mathrm{~S}$.

La serie $\mathrm{B}_{\mathbf{i}}$ dopo la $\mathrm{B}$ cogli stessi liquidi, però stati tolti dalla coppia e poi rimessi.

La $C_{1} 4$ ore e 30 minuti dopo la $B_{\mathbf{4}}$, essendo nell' intervallo la coppia rimasta chiusa con una resistenza esterna $=1$ u. $\mathrm{S}$.

La $\mathrm{D}, 15$ ore dopo, essendo rimasto nell' intervallo il circuito chiuso con resistenza $=1000$.

Le serie $A, B, A_{1}$ furono fatte col metodo di Mance (cioè colla semplice chiusura del solito reoforo $A C$ ); la $B_{1}$ 
col metodo di Mance e con quello di Lodge e si ottennero gli stessi risultati, però con maggior precisione in favore $\mathrm{di}$ quest' ultimo metodo; la $C_{1}$ fu eseguita col metodo di Lodge giacchè quello di Mance non dava alcun effetto se la chiusura di AC era istantanea, mentre se questa reniva prolungata, la diminuzione della forza elettromotrice produceva una diminuzione della deviazione, anche quando col metodo Lodge si otteneva un notevole aumento. La $D_{1}$ fu eseguita col metodo di Lodge.

Riguardo alla sensibilità uno spostamento di 0,02 Siemens nel reocordo produceva nell' ago uno spostanento variabile da 2 a 3 divisioni per le resistenze esterne maggiori (nel qual cuso la seusibilità è maggiore) a 0,4 divisioni per le resistenze esterne $\leq_{4}$ Siemens.

È noterole come la coppia usata per qualche tempo, pur conservando una notevole forza elettromotrice a circuito aperto, ne possieda uno così piccolo a cireuito chiuso da piccole resistenze.

\section{Coppia Volta}

(Zinco in aq. acid. Carbone id. circondato da vaso poroso).

\begin{tabular}{r|c|c}
\hline \multicolumn{3}{c}{$\mathrm{A}$} \\
\hline \hline R oster. & $r$ & $\mathrm{R}$ \\
1000 & 0,21 & 1,56 \\
100 & 0,18 & 1,33 \\
40 & 0,20 & 0,73 \\
4 & 0,18 & 0,33 \\
\hline 10 & 0,22 & 0,29 \\
20 & 0.22 & 0,36 \\
100 & 0,23 & 0,54 \\
1000 & 0,23 & 0,67
\end{tabular}

\begin{tabular}{r|c|c}
\hline \multicolumn{3}{|c|}{ B } \\
\hline R ester. & $r$ & \multicolumn{1}{c}{ E } \\
100 & 0,33 & 0,62 \\
20 & 0,33 & 0,40 \\
5 & 0,34 & 0,29 \\
2 & 0,34 & 0,225 \\
\hline 2 & 0,40 & 0,205 \\
10 & 0,40 & 0,28 \\
50 & 0,39 & 0,41 \\
500 & 0,39 & 0,635 \\
\hline 500 & 0,31 & 0,70 \\
100 & 0,32 & 0,56 \\
40 & 0,32 & 0,33 \\
2 & 0,32 & 0,195
\end{tabular}




\section{6}

La serie $\mathrm{A}$ fu eseg uita con aq. acid. nuora; fra la $1^{2}$ e la $2^{n}$ parte la coppia rimase due ore a circuito chiuso da $R=10$.

La serie B fu eseguita con due coppie collo scopo di anmentare la seusibilità aumentando la differenza di potenziale però essendosi cosi sommate le variazioni delle due coppie, non we risulto un gran vantaggio.

I numeri riferiti danno lo $R_{1} r$ ed $E$ divise per meta per ridurle ad una sola coppia. Al principio della serio le coppie erano rimaste una notte a circuito chiuso da una $\mathrm{R}$ esterna $=200$. Fra lia $1^{\mathrm{a}}$ e la $2^{\mathrm{a}}$ parte il circuito rimase due ore chiuso con $R=4$; fra la $2^{a}$ e la $3^{a}$ parte vi fu un'altra interruzione di circa $\%$ d'ora.

Le variazioni di 0,01 Siem. nel reocordo producevano nell'ago uno spostamento variabile da 1,4 per forti resistenze esterne ad $\frac{1}{\dot{z}}$ di divisione.

Con lieve modificazione, ponendo al luogo della coppia un campione di unità Siemens, e riducendo il sistema di reofori a quello del ponte di Wheatstone, verificai il rapporto di $R$ ad $R_{1}$ che risulto molto prossimamente $=1$.

Si potrà osservare qualche irrogolarità nelle scrie delle resistenze interne e sebbene essa influisca appena sulla seconda cifra decimale, impedisce spesso di scorgere la legge di variazione di essil resistenza colla intensità. La causa di tale irregolarita sta probabilmente nella coppia stessa, in seguito a produzione di bolle di gaz, alterazione dei liquidi, spostamento dei metalli nella coppia.

Per assicurarmi cho essa derivasse veramente da tali cause e non dalla probabile variazione dell' intervallo fra la chiusura del solito reoforo AC e la separazione dell'elettrometro (sebbene non apparisse alcuna decisa relazione fra gli spostamenti dell' ago e la rapidità nella separazione dell' elettrometro) resi le variazioni della resistenza interna della coppia insensibili, aggiungendole una resistenza addizionale di 20 unità, ed allora ottenni per la resistenza totale valori prossimamente identici.

Torino, Fel,braio $18 \Omega 1$.

FINE DEL VOILME IX SERIE TERTI. 
Tuv. I.

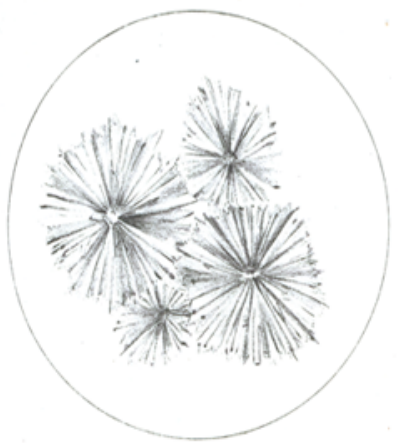

Fig. 2?

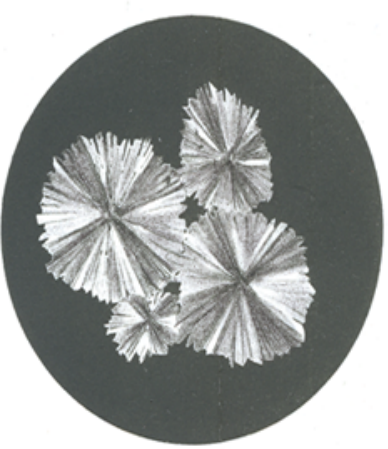

Fịg. $3^{\mathrm{a}}$
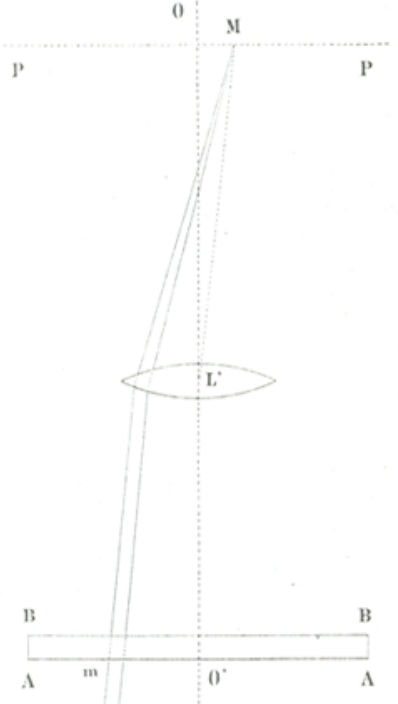

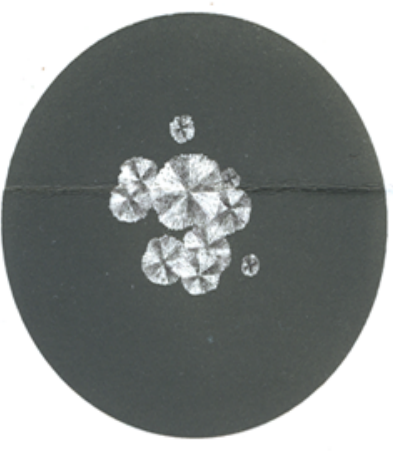

Fig., 4a

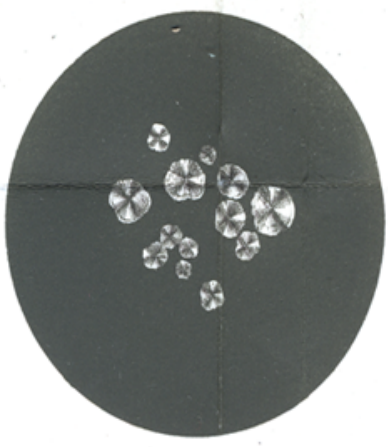

Fig. $5^{\text {a }}$ 


\section{N. Cimento T. IX T. ${ }^{\text {. }}$ II}

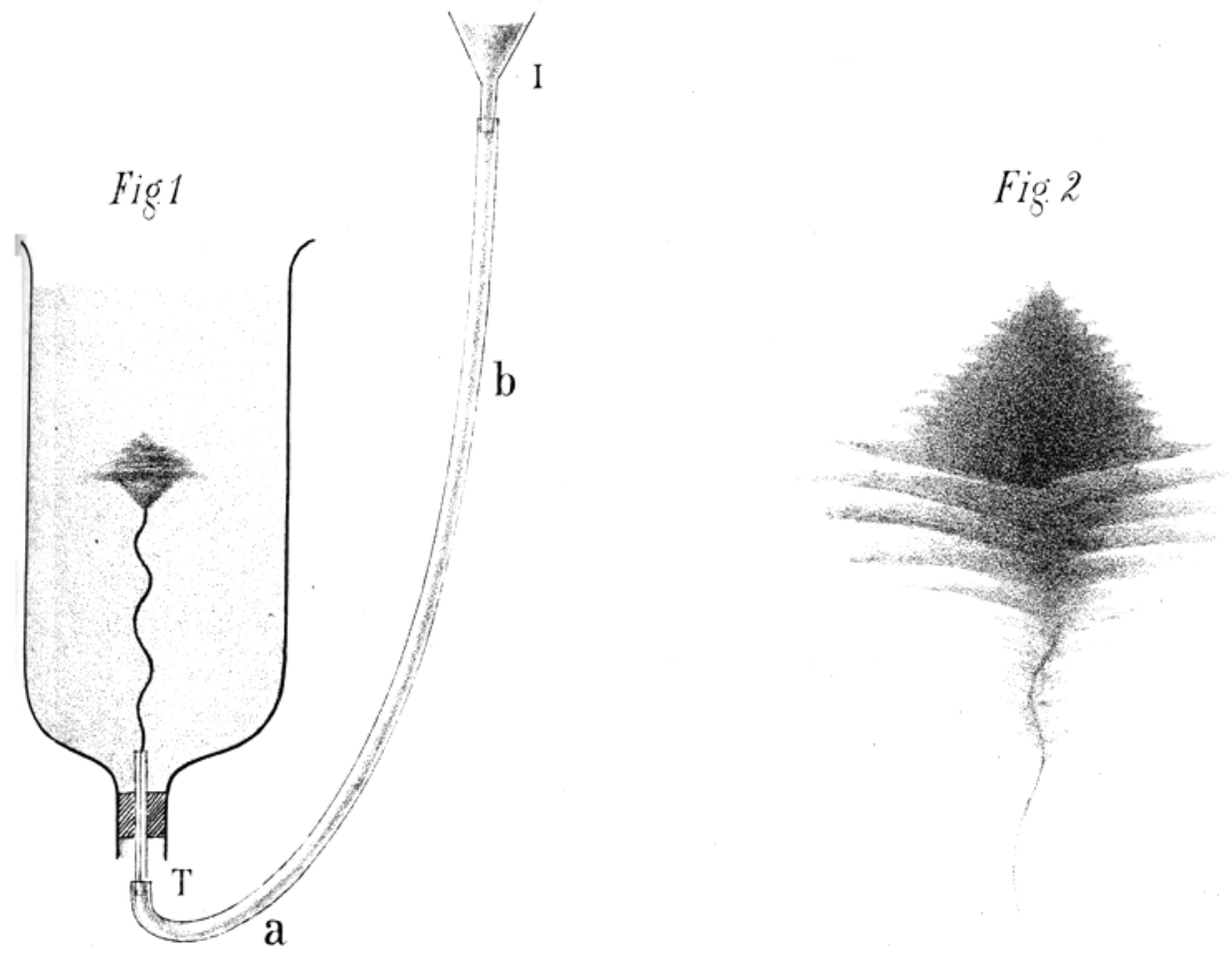

Fig. 3

Figu

R.Lit. Gozani Piśa 


\section{Tav. III.}

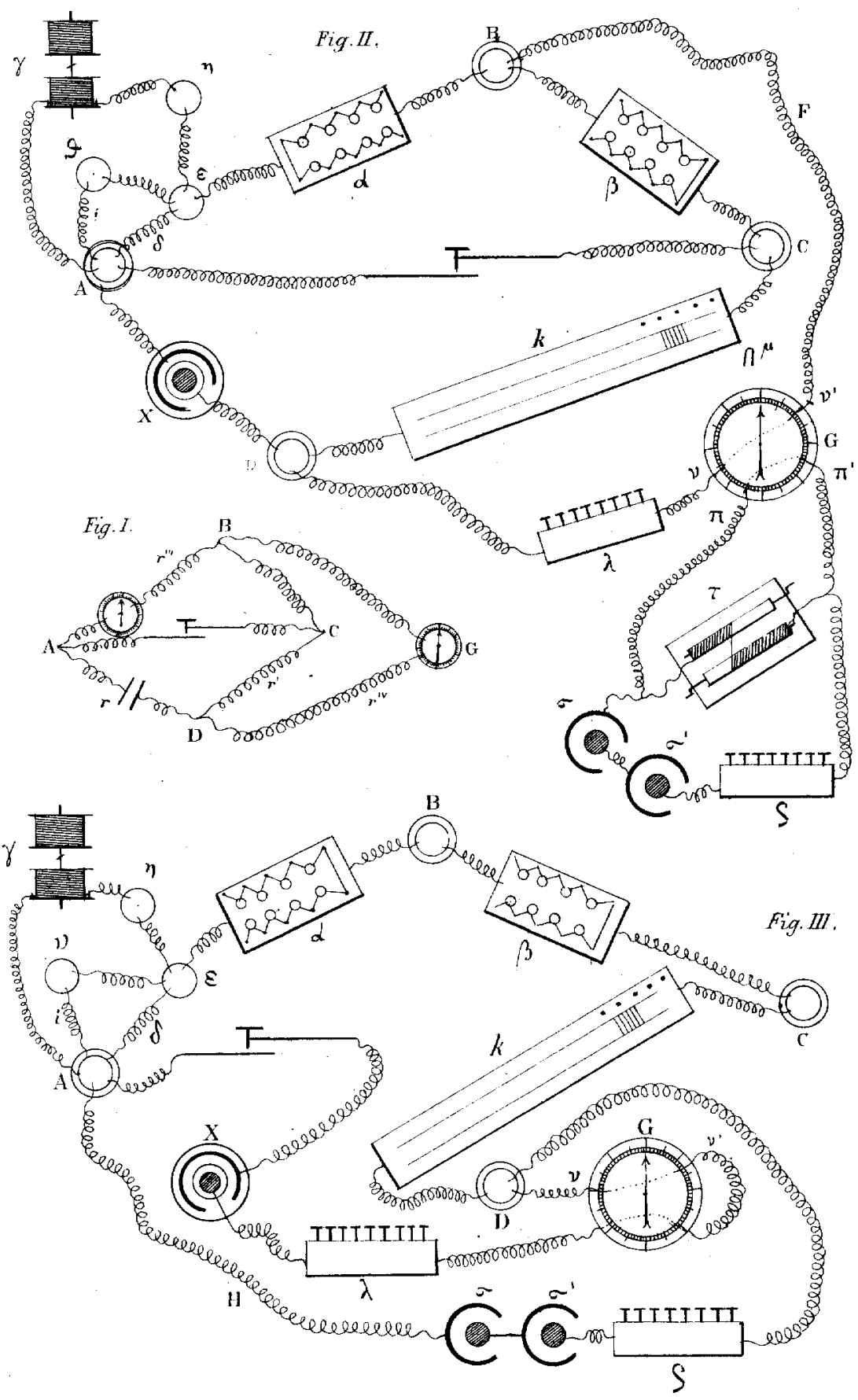


Tav. IV.
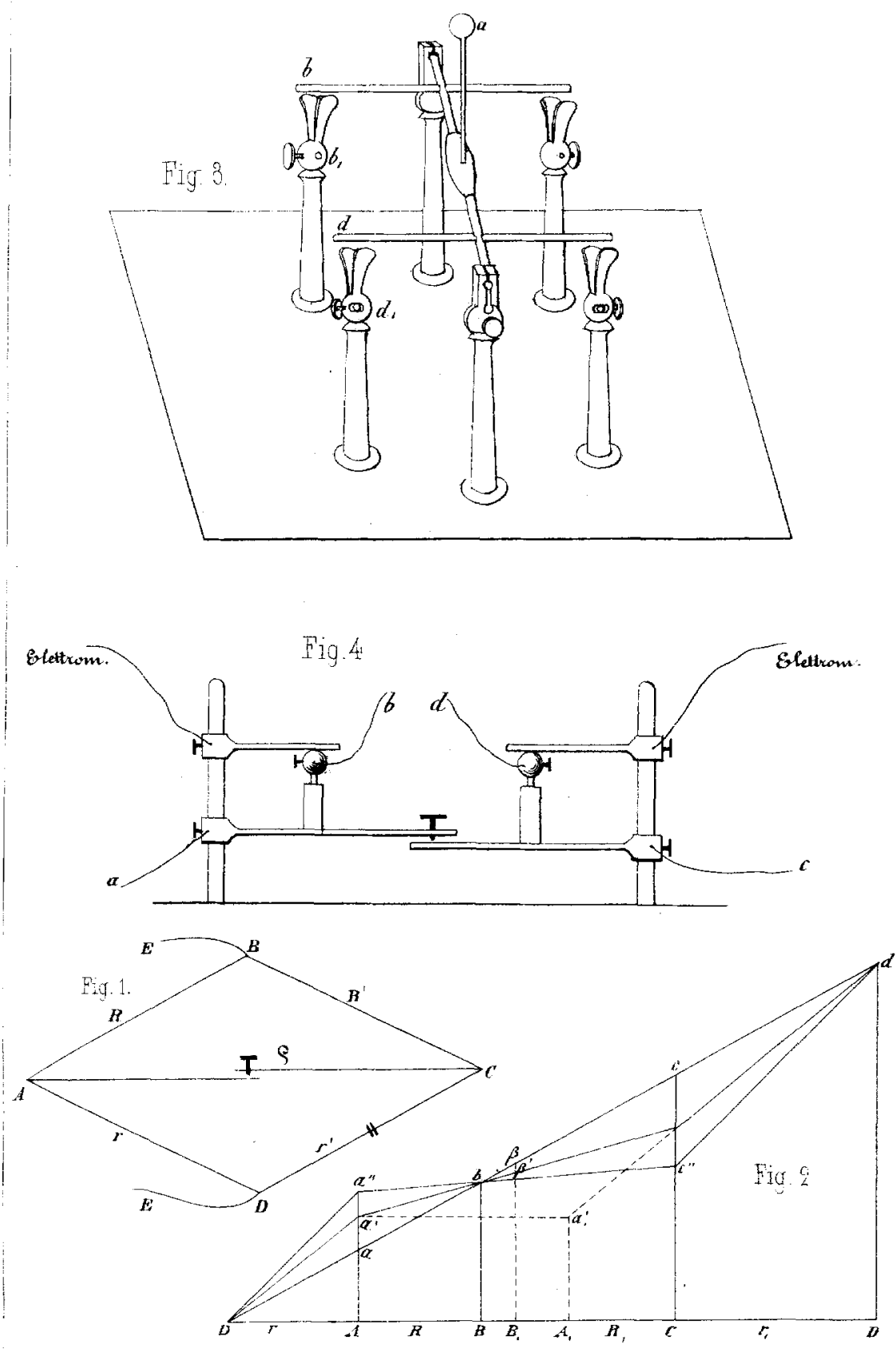